\title{
A REMARK ON THE JET BUNDLES OVER THE PROJECTIVE LINE
}

\author{
INDRANIL BISWAS
}

\section{Introduction}

Let $X$ be a Riemann surface equipped with a projective structure (i.e., a covering by coordinate charts such that the transition functions are of the form $z \longmapsto(a z+b) /(c z+d))$. Let $\mathcal{L}$ be a line bundle on $X$ such that $\mathcal{L}^{2}=T_{X}$. Let $J^{m}\left(\mathcal{L}^{\otimes n}\right) \longrightarrow X$ denote the jet bundle of order $m$ for the line bundle $\mathcal{L}^{\otimes n}$. For $i \geq j$, there is a natural restriction homomorphism from $J^{i}\left(\mathcal{L}^{n}\right)$ onto $J^{j}\left(\mathcal{L}^{n}\right)$. We prove that $J^{n}\left(\mathcal{L}^{n}\right)$ has a natural flat connetion and for any $m \geq n$, the surjective homomorphism

$$
J^{m}\left(\mathcal{L}^{n}\right) \longrightarrow J^{n}\left(\mathcal{L}^{n}\right)
$$

admits a canonical splitting [Theorem 4.1]. As a consequence, for each $n \geq 0$ we construct a differential operator of order $n$ from $\mathcal{L}^{n-1}$ to $\mathcal{L}^{-n-1}$ whose symbol is the constant function 1. Theorem 4.1 follows from the results on the jet bundles over the projective line established in Section 2.

In [CMZ] certain differential operators on a Riemann surface equipped with a projective structure are explicitly constructed (see (3.1)). As an application of the set-up we use to prove Theorem 4.1, in Section 3 we derive the differential operators constructed in [CMZ]. The present work was inspired by [CMZ]; in fact, it grew out of attempts to reconstruct the differential operators there without using the coordinates.

\section{Constructions on the projective line}

Let $V$ be a two dimensional vector space over $\mathbb{C}$. Let $\mathbb{P}(V)$ denote the projective space given by the space of all one dimensional quotients of $V$. Define the line bundle

$$
L:=\mathcal{O}_{\mathbb{P}(V)}(1)
$$

on $\mathbb{P}(V)$, whose fiber over the quotient line $[q]$ is the line $[q]$ itself.

Received May 15, 1996. 
We will recall the definition of the jet bundles for a line bundle. For a line bundle $\xi$ on a Riemann surface $X$, the $n$-th jet bundle, denoted by $J^{n}(\xi)$, is the rank $n+1$ vector bundle on $X$ whose fiber over $x \in X$ is

$$
\left(\mathcal{O}_{X, x} / m_{x}^{n+1}\right) \otimes_{\mathbb{C}} \xi_{x}
$$

where $\xi_{x}$ is the fiber of $\xi$ over $x$, and $\mathcal{O}_{X, x}$ is the ring of functions defined around $x$ with $m_{x}$ being the maximal ideal consisting of functions vanishing at $x$. The inclusion of $m_{x}^{n+1}$ in $m_{x}^{n}$ induces the following short exact sequence of vector bundle on $X$ :

$$
0 \longrightarrow K_{X}^{\otimes n} \otimes \xi \longrightarrow J^{n}(\xi) \longrightarrow J^{n-1}(\xi) \longrightarrow 0
$$

where $K_{X}$ is the canonical bundle of $X$.

Let $\mathcal{V}$ denote the rank two trivial vector bundle on $\mathbb{P}(V)$ with $V$ as the fiber. For any $n \geq 0, S^{n}(\mathcal{V})$ will denote the $n$-th symmetric power of $\mathcal{V}$, with $S^{0}(\mathcal{V})$ being the trivial line bundle.

Lemma 2.2. For any integer $n \geq 0$, the vector bundle $J^{n}\left(L^{n}\right)$ on $\mathbb{P}(V)$ is canonically isomorphic to the symmetric power $S^{n}(\mathcal{V})$. For any $m \geq n$, the surjection

$$
J^{m}\left(L^{n}\right) \longrightarrow J^{n}\left(L^{n}\right) \longrightarrow 0
$$

given by (2.1), admits a canonical splitting (i.e., a homomorphism from $J^{n}\left(L^{n}\right)$ to $J^{m}\left(L^{n}\right)$ such that the composition is identity on $J^{n}\left(L^{n}\right)$.

Proof. Take any integer $n \geq 0$. Since $S^{n}(V)=H^{0}\left(\mathbb{P}(V), L^{n}\right)$, for any $x \in \mathbb{P}(V)$ there is a natural homomorphism of $S^{n}(V)$ into the fiber $J^{n}\left(L^{n}\right)_{x}$ given by the restriction of sections to the $n$-th order infinitesimal neighborhood of $x$. Since for any integer $j$ with $j \leq n$,

$$
\begin{aligned}
& \operatorname{dim} H^{0}\left(\mathbb{P}(V), L^{n} \otimes \mathcal{O}_{\mathbb{P}(V)}(-j x)\right)- \\
& \operatorname{dim} H^{0}\left(\mathbb{P}(V), L^{n} \otimes \mathcal{O}_{\mathbb{P}(V)}(-(j+1) x)\right)=1
\end{aligned}
$$

the above obtained homomorphism must be an isomorphism. This proves the first part of the lemma.

Take any integer $m$ such that $m \geq n$. We may restrict a section of $L^{n}$ to the $m$-th order infinitesimal neighborhood of $x$ to get a homomorphism from the vector space $S^{n}(V)\left(=H^{0}\left(\mathbb{P}(V), L^{n}\right)\right)$ to the fiber $J^{m}\left(L^{n}\right)_{x}$. Now using the previous identification of $S^{n}(V)$ with $J^{n}\left(L^{n}\right)_{x}$ we get the required splitting.

Setting $m=n+1$ in Lemma 2.2 we obtain the following:

Corollary 2.3. For any integer $n \geq 0$, the exact sequence

$$
0 \longrightarrow K_{\mathbb{P}(V)}^{n+1} \otimes L^{n} \longrightarrow J^{n+1}\left(L^{n}\right) \longrightarrow J^{n}\left(L^{n}\right) \longrightarrow 0
$$

admits a canonical splitting. 
Choose and fix a trivialization of $\wedge^{2} V$; this is equivalent to fixing a nonzero vector $\theta$ in $\stackrel{2}{\wedge} V$. The canonical bundle $K_{\mathbb{P}(V)}=L^{-2} \otimes \operatorname{det} \mathcal{V}$. Using the trivialization of $\stackrel{2}{\wedge} V$ we have, $K_{\mathbb{P}(V)}=L^{-2}$.

The sheaf of differential operators of order $k$ from the sections of a line bundle $\xi$ to the sections of a line bundle $\eta$ is precisely the sheaf $\operatorname{Hom}\left(J^{k}(\xi), \eta\right)$. Consider the projection of $J^{n+1}\left(L^{n}\right)$ onto $K_{\mathbb{P}(V)}^{n+1} \otimes L^{n}=$ $L^{-n-2}$ defining the splitting in Corollary 2.3. This gives a global differential operator of order $n+1$,

$$
\mathcal{D}(n+1) \in H^{0}\left(\mathbb{P}(V), \operatorname{Diff}^{n+1}\left(L^{n}, L^{-n-2}\right)\right) .
$$

The symbol of a differential operator in $\operatorname{Diff}^{n+1}\left(L^{n}, L^{-n-2}\right)$ is a section of the line bundle $T_{\mathbb{P}(V)}^{n+1} \otimes L^{-2 n-2}=\mathcal{O}_{\mathbb{P}(V)}$. Since the differential operator $\mathcal{D}(n+1)$ in (2.4) gives a splitting of the jet sequence - it's symbol, which is a constant function, must be the constant function 1 .

Let $S L(V)$ denote the subgroup of $G L(V)=\operatorname{Aut}(V)$ that acts trivially on $\stackrel{2}{\wedge} V$. The group $S L(V)$ has a natural action on $\mathbb{P}(V)$, and $\operatorname{Aut}(\mathbb{P}(V))=$ $S L(V) / \mathbb{Z}_{2}$. There is a natural induced action of $S L(V)$ on any sheaf $J^{m}\left(L^{n}\right)$ that lifts the action on $\mathbb{P}(V)$. The isomorphism between $S^{n}(\mathcal{V})$ and $J^{n}\left(L^{n}\right)$, and the splitting in Lemma 2.2, are both equivariant for this action. Indeed, this follows from the canonical nature of the construction in Lemma 2.2. So, in particular, the differential operator $\mathcal{D}(n)$ in $(2.4)$ is an invariant for the action of $S L(V)$ on the space of all global sections of $\operatorname{Diff}^{n}\left(L^{n-1}, L^{-n-1}\right)$.

Note that $\mathcal{D}(n)$ is not an invariant for the action $G L(V)$ since the trivialization of ${ }^{\wedge} V$ was used in its construction. The identification between $K_{\mathbb{P}(V)}$ and $L^{-2}$ is not equivariant for the action of the center of $G L(V)$.

Setting $n=2$ in Lemma 2.2 we get that $J^{2}\left(L^{2}\right)=S^{2}(\mathcal{V})$. This implies that the homomorphism

$$
\rho: H^{0}\left(\mathbb{P}(V), J^{2}\left(L^{2}\right)\right) \longrightarrow H^{0}\left(\mathbb{P}(V), L^{2}\right)
$$

induced by the obvious projection, namely $J^{2}\left(L^{2}\right) \longrightarrow L^{2}$, is actually an isomorphism. Moreover, $\rho$ is the identity map of $S^{2}(V)$. Thus, after identifying the tangent bundle $T_{\mathbb{P}(V)}$ with $L^{2}$ using the trivialization of $\stackrel{2}{\wedge} V$, the Lemma 2.2 implies that

$$
H^{0}\left(\mathbb{P}(V), T_{\mathbb{P}(V)}\right)=H^{0}\left(\mathbb{P}(V), J^{2}\left(L^{2}\right)\right)=S^{2}(V) .
$$

The Lie-bracket operation equips the vector space $H^{0}\left(\mathbb{P}(V), T_{\mathbb{P}(V)}\right)$ with the structure of a Lie algebra. The action of $S L(V)$ on $\mathbb{P}(V)$ gives a Lie algebra homomorphism from its Lie algebra, $s l(V)$, into $H^{0}\left(\mathbb{P}(V), T_{\mathbb{P}(V)}\right)$. 
This homomorphism is actually an isomorphism. The Lie algebra structure on $S^{2}(V)$ induced by the equality $(2.5)$ can be seen directly as follows: using contraction, $S^{2}(V)$ maps $V^{*}$ into $V$; on the other hand, $\theta$ identifies $V^{*}$ with $V$ - combining these, the resulting homomorphism from $S^{2}(V)$ into $\operatorname{sl}(V)$ is an isomorphism.

Let $C \in S^{2}\left(H^{0}\left(\mathbb{P}(V), T_{\mathbb{P}(V)}\right)\right)$ be the Casimir of the Lie algebra $H^{0}\left(\mathbb{P}(V), T_{\mathbb{P}(V)}\right)$. The section $C$ is evidently an invariant for the obvious action of $S L(V)$ on the vector space $S^{2}\left(H^{0}\left(\mathbb{P}(V), T_{\mathbb{P}(V)}\right)\right)$. For a section $s$ of $T_{\mathbb{P}(V)}$, let $L_{s}$ denote the Lie derivative with respect to $s$. The (second order) Lie derivative with respect to $s \otimes s$ is defined to be $L_{s} \circ L_{s}$. Thus $C$ acts as a differential operator, denoted by $L_{C}$, on all vector bundles associated to $\mathbb{P}(V)$. This differential operator is actually of order zero (i.e., a constant scalar multiplication).

Using (2.5) and Lemma 2.2 we get that

$$
S^{2}\left(H^{0}\left(\mathbb{P}(V), T_{\mathbb{P}(V)}\right)\right)=H^{0}\left(\mathbb{P}(V), S^{2}\left(J^{2}\left(T_{\mathbb{P}(V)}\right)\right)\right) .
$$

Let $\bar{C} \in H^{0}\left(\mathbb{P}(V), S^{2}\left(J^{2}\left(T_{\mathbb{P}(V)}\right)\right)\right)$ be the element corresponding to the Casimir $C$; $\bar{C}$ is actually the Casimir for the Lie algebra $S^{2}(V)$ (which is the fiber of $J^{2}\left(T_{\mathbb{P}(V)}\right)$.

Let $p: J^{2}\left(T_{\mathbb{P}(V)}\right) \longrightarrow T_{\mathbb{P}(V)}$ be the obvious projection. If (locally)

$$
\bar{C}=\sum_{i} A_{i} \otimes A_{i}
$$

where $A_{i}$ are local sections of $J^{2}\left(T_{\mathbb{P}(V)}\right)$, consider the operator

$$
L_{\bar{C}}=\sum L_{p\left(A_{i}\right)} \circ L_{p\left(A_{i}\right)}
$$

with $L_{\phi\left(A_{i}\right)}$ being the Lie derivative with respect to the vector field $p\left(A_{i}\right)$. It is easy to check that the operator $L_{\bar{C}}$ does not depend upon the choice of the decomposition of $\bar{C}$, and that $L_{C}=L_{\bar{C}}$.

\section{Jets of the trivial line bundle on the projective line}

Let $\operatorname{Diff}^{n}(\mathcal{O}, \mathcal{O})=J^{n}(\mathcal{O})^{*}$ be the sheaf of differential operators on the trivial line bundle over $\mathbb{P}(V)$. The symbol map, which is the dual of the injection in (2.1), gives a surjective homomorphism

$$
\sigma: \operatorname{Diff}^{n}(\mathcal{O}, \mathcal{O}) \longrightarrow T_{\mathbb{P}(V)}^{n} .
$$

Let $\gamma$ denote the obvious projection of $J^{n-1}\left(T_{\mathbb{P}(V)}^{n}\right)$ onto $T_{\mathbb{P}(V)}^{n}$.

For any $n \geq 1$, let $J_{0}^{n}(\mathcal{O}) \subset J^{n}(\mathcal{O})$ be the kernel of the obvious homomorphism from $J^{n}(\mathcal{O})$ onto $J^{0}(\mathcal{O})=\mathcal{O}$. This subsheaf has a canonical splitting given by the constant functions. Define the subsheaf, $\operatorname{Diff}_{0}^{n}(\mathcal{O}, \mathcal{O}):=J_{0}^{n}(\mathcal{O})^{*}$, of $\operatorname{Diff}^{n}(\mathcal{O}, \mathcal{O})$. 
For a function $f$ on $\mathbb{C}$ and any integer $n \geq 1$, in Proposition 1 (page 4 ) of [CMZ] (where it is called $\mathcal{L}_{-n}(f)$ ) the following differential operator of order $n$ is constructed:

$$
D_{n}(f):=\sum_{i=0}^{n-1} \frac{(2 n-i) !}{i !(n-i) !(n-i-1) !} f^{(i)} \partial^{n-i}
$$

with $f^{(i)}=\partial^{i} f$ being the $i$-th derivative of $f$. The operator $D_{n}$ has the property that for any Möbius transformation, $M(z)=(a z+b) /(c z+d)$, of $\mathbb{C P}^{1}$, the following equality holds:

$$
D_{n}(f) \circ M=D_{n}\left(M_{n} \cdot(f \circ M)\right)
$$

where $M_{n}(z)=(c z+d)^{2 n}$.

Thus $D_{n}$ is a $S L(V)$ equivariant $\mathcal{O}_{\mathbb{P}(V)}$ linear isomorphism (in other words, a canonical isomorphism)

$$
\phi: J^{n-1}\left(T_{\mathbb{P}(V)}^{n}\right) \longrightarrow \operatorname{Diff}_{0}^{n}(\mathcal{O}, \mathcal{O}) .
$$

The operator $D_{n}$ has the further property that $\sigma \circ \phi=\gamma$. It is shown in [CMZ] that this splitting condition together with the automorphic property (3.2) actually determine the operator $D_{n}$. In this section we want to deduce the above result of [CMZ] in the set-up of Section 2.

Take a point $x \in \mathbb{P}(V)$. The long exact sequence of cohomology for the exact sequence of sheaves on $\mathbb{P}(V)$

$$
0 \longrightarrow \mathcal{O}(-(n+1) \cdot x) \longrightarrow \mathcal{O} \longrightarrow J^{n}(\mathcal{O})_{x} \longrightarrow 0
$$

gives the equality

$$
J_{0}^{n}(\mathcal{O})_{x}=H^{1}(\mathbb{P}(V), \mathcal{O}(-(n+1) \cdot x))
$$

where $J_{0}^{n}(\mathcal{O})_{x}$ is the fiber of $J_{0}^{n}(\mathcal{O})$ over $x$.

Choose and fix an isomorphism between the two line bundles $\mathcal{O}(x)$ and $L$. Since the fiber $\mathcal{O}(x)_{x}=T_{\mathbb{P}(V), x}=L_{x}^{2}$, fixing such an isomorphism is equivalent to fixing a nonzero vector $\omega$ in $L_{x}$.

Using Serre duality for $\mathcal{O}(-(n+1) \cdot x)$, and then identifying $K_{\mathbb{P}(V)}$ with $\mathcal{O}(-2 x)$ using $\omega$, we have

$$
\begin{aligned}
& \operatorname{Diff}_{0}^{n}(\mathcal{O}, \mathcal{O})_{x}= \\
& H^{0}(\mathbb{P}(V), \mathcal{O}((n-1) \cdot x))=H^{0}\left(\mathbb{P}(V), L^{n-1}\right)=S^{n-1}(V) .
\end{aligned}
$$

Consider the restriction of sections of $T_{\mathbb{P}(V)}^{n}$ to the $(n-1)$-th order infinitesimal neighborhood of $x$, namely

$$
\beta: S^{2 n}(V)=H^{0}\left(\mathbb{P}(V), T_{\mathbb{P}(V)}^{n}\right) \longrightarrow J^{n-1}\left(T_{\mathbb{P}(V)}^{n}\right)_{x}
$$


which is clearly a surjective homomorphism. Indeed, in the proof of Lemma 2.2 we saw that $S^{2 n}(V)$ surjects onto $J^{2 n}\left(L^{2 n}\right)_{x}=J^{2 n}\left(T_{\mathbb{P}(V)}^{n}\right)_{x}$. We want to identify the kernel of the homomorphism $\beta$.

The symplectic form on $V$ given by the trivialization of ${ }^{2} V$ identifies $V$ with $V^{*}$. Let $v$ be the vector in the kernel of the quotient homomorphism $V \longrightarrow L_{x}$ which corresponds to $\omega$ by the isomorphism between $V$ and $V^{*}$ given by the symplectic form on $V$. (This vector $v \in V$ corresponds to the section of the sheaf $\mathcal{O}(x)$ given by the constant function 1.)

Consider the homomorphism, $m_{v}: S^{n}(V) \longrightarrow S^{2 n}(V)$, defined by multiplication with $v^{\otimes n}$. The inclusion $m_{v}$ corresponds to the natural inclusion of $H^{0}(\mathbb{P}(V), \mathcal{O}(n . x))$ into $H^{0}(\mathbb{P}(V), \mathcal{O}(2 n . x))$. The image of $m_{v}$ is precisely the kernel of $\beta$ in (3.5).

Consider the homomorphism $i_{\omega}: S^{2 n}(V) \longrightarrow S^{n-1}(V)$ given by the contraction with $\omega^{\otimes(n+1)}$. (The vector $\omega$ is considered as an element of $V^{*}$.) This homomorphism vanishes on the image $m_{v}\left(S^{n}(V)\right)$. Indeed, this follows from the fact that $\omega(v)=0$.

Thus using the equality (3.4) and $i_{\omega}$ we have the homomorphism

$$
\phi_{x}: J^{n-1}\left(T_{\mathbb{P}(V)}^{n}\right)_{x} \longrightarrow \operatorname{Diff}_{0}^{n}(\mathcal{O}, \mathcal{O})_{x} .
$$

It is easy to check that the homomorphism $\phi_{x}$ does not depend upon the choice of the nonzero vector $\omega \in L_{x}$. The resulting homomorphism $\phi$ from $J^{n-1}\left(T_{\mathbb{P}(V)}^{n}\right)$ to $\operatorname{Diff}_{0}^{n}(\mathcal{O}, \mathcal{O})$ satisfies the condition that $\sigma \circ \phi=\gamma$. The canonical nature of the construction of $\phi$ ensures that it is equivariant for the action of $S L(V)$. Since $\phi_{x}$ is an isomorphism, $\phi$ is an isomorphism.

Let $U \subset S L(V)$ be the unipotent subgroup which fixes the vector $v$. Let $\mathfrak{n}$ be the nilpotent part of the Lie algebra of $U$. Let $N$ denote the unique element in $\mathfrak{n}$ which maps a preimage of $\omega$ (in $V$ ) to $v$. For any $0 \leq i \leq 2 n$, the image of $S^{i}(V)$ in $S^{2 n}(V)$, for the homomorphism given by the multiplication with $v^{2 n-i}$, is denoted by $S_{i}^{2 n}(V)$. In this notation, $N$ maps $S_{i+1}^{2 n}(V)$ onto $S_{i}^{2 n}(V)$; the resulting homomorphism from $S^{i+1}(V)$ onto $S^{i}(V)$ is the contraction by $\omega$. From this it is easy to deduce that any homomorphism from $S^{2 n}(V) / m_{v}\left(S^{n}(V)\right)$ to $S^{n-1}(V)$, which is equivariant for the actions of $N$, must be a scalar multiple of $i_{\omega}$. Now the condition, $\sigma \circ \phi=\gamma$, uniquely determines the homomorphism $\phi$.

\section{Jets on a Riemann surface with a projective structure}

Let $X$ be a Riemann surface, not necessarily compact. A projective structure on $X$ is a maximal atlas of holomorphic coordinate charts, $\left\{U_{\alpha}, f_{\alpha}\right\}_{\alpha \in I}$, covering $X$, such that any $f_{\alpha}$ maps $U_{\alpha}$ biholomorphically onto some analytic open set in $\mathbb{P}(V)$ and the transition function $f_{\alpha} \circ f_{\beta}^{-1}$, for any $\alpha, \beta \in I$, is a restriction of an automorphism of $\mathbb{P}(V),[\mathrm{G}],[\mathrm{D}],[\mathrm{T}]$. 
We note that any Riemann surface admits a projective structure, since, from the uniformization theorem, the universal cover has a natural projective structure. It is know that for any projective structure, it is possible to choose a sub-cover such that the transition functions have a compatible lift to $S L(V)$ (from $\operatorname{Aut}(\mathbb{P}(V)))$. Actually, more than one inequivalent lifts are possible. For a compact Riemann surface, the set of equivalence classes of lifts correspond to the set of square roots of the canonical bundle (called theta characteristics) $[\mathrm{G}],[\mathrm{T}]$. Henceforth, by a projective structure we will always mean a lift of the structure group to $S L(V)$.

Let $X$ be a Riemann surface equipped with a projective structure in the above sense.

Since the natural action of $S L(V)$ on $\mathbb{P}(V)$ lifts to the bundle $L$, the projective structure gives a line bundle on $X$ associated to $L$. Let $\mathcal{L}$ denote this line bundle on $X$. Since the isomorphism between $L^{2}$ and $T_{\mathbb{P}(V)}$ is $S L(V)$ equivariant, we have $\mathcal{L}^{\otimes 2}=T_{X}$.

Since $J^{n}\left(L^{n}\right)$ on $\mathbb{P}(V)$ is a trivial bundle (Lemma 2.2), it has a natural flat connection, which is equivariant under the action of $S L(V)$. We now have the following consequence of Lemma 2.2, Corollary 2.3 and (2.4):

Theorem 4.1. For any $n \geq 0$, the jet bundle $J^{n}\left(\mathcal{L}^{n}\right)$ on $X$ has a natural flat connection, and $S^{n}\left(J^{1}(\mathcal{L})\right)=J^{n}\left(\mathcal{L}^{n}\right)$, with the identification being compatible with the flat connections. For any $m \geq n$, the natural surjection

$$
J^{m}\left(\mathcal{L}^{n}\right) \longrightarrow J^{n}\left(\mathcal{L}^{n}\right) \longrightarrow 0
$$

has a canonical splitting. Setting $m=n+1$, a global differential operator of order $n+1$

$$
\mathcal{D}_{X}(n+1) \in H^{0}\left(X, \operatorname{Diff}_{X}^{n}\left(\mathcal{L}^{n}, \mathcal{L}^{-n-2}\right)\right)
$$

is obtained. The symbol of $\mathcal{D}_{X}(n)$ is the constant function 1 . The fibers of $J^{2}\left(T_{X}\right)$ have the structure of a Lie algebra compatible with the flat connection on $J^{2}\left(T_{X}\right)$. The Lie derivative action of the Casimir

$$
C_{X} \in H^{0}\left(X, S^{2}\left(J^{2}\left(T_{X}\right)\right)\right)
$$

on any tensor power of $\mathcal{L}$ is a multiplication by a constant scalar.

Similarly, since the isomorphism $\phi$ in $(3.3)$ is $S L(V)$ equivariant, we have an isomorphism of vector bundles on $X$

$$
\phi_{X}: J^{n-1}\left(T_{X}^{n}\right) \longrightarrow \operatorname{Diff}_{0}^{n}(\mathcal{O}, \mathcal{O})
$$

$\left(\operatorname{Diff}_{0}^{n}(\mathcal{O}, \mathcal{O}) \subset \operatorname{Diff}_{X}^{n}(\mathcal{O}, \mathcal{O})\right.$ is the canonical complement of $\left.\operatorname{Diff}_{X}^{0}(\mathcal{O}, \mathcal{O})\right)$ such that the composition of the symbol map on $\operatorname{Diff}_{X}^{n}(\mathcal{O}, \mathcal{O})$ with the isomorphism $\phi_{X}$ is the natural projection of $J^{n-1}\left(T_{X}^{n}\right)$ onto $T_{X}^{n}$. 


\section{References}

[CMZ] P. Cohen, Y. Manin, and D. Zagier, Automorphic pseudodifferential operators, MPI Preprint (1995).

[D] P. Deligne, Equations Différentielles à Points Singuliers Réguliers, Lecture Notes in Math. 163 (1970), Springer-Verlag, Berlin-Heidelberg-New York.

[G] R. C. Gunning, Lectures on Riemann surfaces, Math. Notes 2, Princeton University Press, Princeton, New Jersey 1966.

[T] A. N. Tyurin, On periods of quadratic differentials, Russian Math. Surveys 33 (1978), 169-221.

School of Mathematics, Tata Institute of Fundamental Research, Homi BhabHa Road, Bombay 400005, INDIA

E-mail address: indranil@math.tifr.res.in 